\title{
Assistência de enfermagem ao paciente com suspeita de neoplasia em cabeça/pescoço: relato de experiência
}

RESUMO | Objetivo: Relatar diante da história clínica deste paciente dos sinais sintomas apresentados, diagnóstico médico e tratamento, apresentar quais os cuidados de enfermagem necessários ao paciente com suspeita de neoplasia de cabeça e pescoço. Método: Trata-se de um estudo descritivo de abordagem qualitativa, do tipo relato de experiência. Para a realização do estudo foram utilizadas informações do prontuário do paciente e histórico de enfermagem. O estudo foi aprovado por Comitê de Ética. Resultado: Dentre os cuidados de enfermagem, cita-se: Realizar balanço hídrico, manter cabeceira elevada, auxiliar na alimentação, orientar sobre a doença, entre outros. Através da vivência foi possível observar a importância de cuidados específicos para o paciente, através da aplicação processo de enfermagem. Conclusão: Em vista disso concluímos que a assistência de enfermagem prestada ao paciente com câncer cabeça e pescoço são essenciais para a qualidade do cuidado e para sua efetiva recuperação. Neste sentido o enfermeiro deve se fazer presente como um profissional de referência por meio de conhecimento técnico e baseado em evidência. Palavras-chaves: Neoplasias; Enfermagem; Tabagismo; Alcoolismo.

\begin{abstract}
Objective: To report, in view of the clinical history of this patient, of the signs and symptoms presented, medical diagnosis and treatment, to present what nursing care is necessary for the patient with suspected head and neck cancer. Method: This is a descriptive study with a qualitative approach, an experience report type. To carry out the study, information from the patient's medical record and nursing history were used. The study was approved by the Ethics Committee. Result: Among the nursing care, the following are cited: Perform water balance, keep the headboard elevated, assist in feeding, advise on the disease, among others. Through the experience it was possible to observe the importance of specific care for the patient, through the application of the nursing process. Conclusion: In view of this, we conclude that the nursing care provided to patients with head and neck cancer are essential for the quality of care and for their effective recovery. In this sense, the nurse must be present as a reference professional through technical knowledge and based on evidence.
\end{abstract}

Keywords: Neoplasms; Nursing; Smoking; Alcoholism.

RESUMEN | Objetivo: Describir, a la vista de la historia clínica del paciente, signos, síntomas, diagnóstico médico y tratamiento evidenciados, presentar los cuidados de enfermería necesarios para los pacientes con sospecha de cáncer de cabeza y cuello. Método: Se trata de un estudio descriptivo con enfoque cualitativo, del tipo relato de experiencia. Para la realización del estudio se utilizó información de la historia clínica del paciente y del historial de enfermería. El estudio fue aprobado por el Comité de Ética. Resultado: Entre los cuidados de enfermería se citan los siguientes: Realizar balance hídrico, mantener elevado el cabecero, asistir en la alimentación, asesorar sobre la enfermedad, entre otros. A través de la experiencia, fue posible observar la importancia del cuidado específico para el paciente, a través de la aplicación del proceso de enfermería. Conclusión: Ante esto, se concluye que los cuidados de enfermería que se brindan a los pacientes con cáncer de cabeza y cuello son fundamentales para la calidad de la atención y para su efectiva recuperación. En este sentido, el enfermero debe estar presente como un profesional de referencia a través de conocimientos técnicos y con base en la evidencia.

Palabras claves: Neoplasias; Enfermería; De fumar; Alcoholismo.

Eva Natalina Ferreira Costa

Residente de Enfermagem pela Universidade Federal do Estado do Rio de Janeiro (UNIRIO). ORCID: 0000-0003-2673-6967

\section{Flávia Martins Branco}

Residente de Enfermagem pela Universidade Federal do Estado do Rio de Janeiro (UNIRIO). ORCID: 0000-0003-3484-8555

\section{Dayane Martins da Silva Campos}

Residente de Enfermagem pela Universidade Federal do Estado do Rio de Janeiro (UNIRIO). ORCID: 0000-0002-0193-4417

Recebido em: 11/02/2021

Aprovado em: 14/05/2021
INTRODUÇÃO

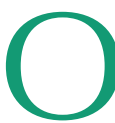
Câncer é a doença que mais preocupa a população nos últimos tempos, é o nome dado a um conjunto de mais de 100 doenças que têm em comum o crescimento desordenado de células, que invadem tecidos e órgãos. Dividindo-se rapidamente, essas células tendem a ser muito agressivas e incontroláveis, determinando a formação de tumores, que podem espaIhar-se para outras regiões do corpo'.

O câncer é um problema de saúde pública no mundo e encontra se entre as quatro principais causas de mortes prematuras (antes dos 70 anos de idade) na maioria dos países. A incidência e a mortalidade pela doença vêm aumentando no mundo, em parte pelo aumento da perspectiva de vida, pelo crescimento populacional, como também pela variação na distribuição e na prevalência dos fatores de risco de câncer, especialmente aos associados ao desenvolvimento socioeconômico².

Um dos fatores que mais agravam a ocorrência do câncer Cabeça e Pescoço (CCP) é a falta de informação. Cerca de $70 \%$ dos pacientes no Brasil são diagnos- 
ticados com a doença em estado avançado, o que dificulta a possibilidade de cura deste tipo de câncer. Quando o diagnóstico é realizado no estágio inicial as possibilidades de cura chega a $80 \%{ }^{3}$.

Os principais fatores de risco associados para o surgimento dessa doença é o tabagismo e o etilismo, aumentando em até 20 vezes a chance de uma pessoa saudável vir a desenvolver a patologia, pois $75 \%$ a $90 \%$ dos casos estão diretamente relacionados com estes agentes carcinogênicos. Cita-se ainda, os maus hábitos alimentares e infecções virais, como o Papiloma Vírus Humano, também estão associados a doença ${ }^{4}$.

Ao ser diagnosticado, o paciente com câncer de cabeça e pescoço confronta as mais diversas dificuldades em decorrência dessa nova condição. Dentre elas, inclui-se o isolamento social, baixa na autoestima, alterações físicas, fisiológicas e psicológicas, perda da autonomia para o seu autocuidado com o avançar do quadro, sofrimento relacionado ao tratamento, aspectos sociais, culturais e psicossociais e mudanças dos hábitos alimentares, tais fatores geram grande sensibilização para o paciente e a família ${ }^{5}$.

Uma lesão na boca, nódulos no pescoço, rouquidão prolongada, dificuldades de engolir são sintomas a se atentar, pois são indicativos para o CCP. Esses tumores atingem a cavidade oral, orofaringe, nasofaringe, hipofaringe, laringe, cavidade nasal, seios paranasais, glândulas salivares, essas regiões são responsáveis por funções como deglutição, fala, respiração, paladar e olfato. Todos estes sintomas são característicos da doença, mas muitas vezes não são levados em consideração pelo indivíduo ${ }^{6}$.

Diante das diversas necessidades do cuidado com o paciente com câncer cabeça e pescoço torna se relevante, o trabalho realizado pela equipe interdisciplinar de saúde sobre os fatores subjetivos do indivíduo e da conexão entre equipe paciente e família por meio da comunicação ativa e efetiva ${ }^{7}$.
O processo de enfermagem inclui coleta de dados, diagnóstico, planejamento, intervenção e avaliação. O enfermeiro tem papel fundamental na assistência, ao coletar os dados de um paciente ele processam em informações, em seguida organizam em categorias de conhecimento. A coleta de dados proporciona a melhor oportunidade para enfermeiros estabelecer uma relação terapêutica real com o paciente $^{8}$.

As questões culturais envolvidas no cuidado às pessoas com CCP podem ser compreendidas e interpretadas, sendo assim para uma boa relação entre a equipe, paciente e família, os profissionais de enfermagem tem como base uma excelente comunicação. Torna se impraticável, ao avaliar a atividades complexas exercidas pela enfermagem, com dependência da comunicação com a possibilidade executar suas atribuições sem se comunicar com as pessoas envolvidas neste cenário ${ }^{9}$.

O objetivo deste relato de experiência é diante da história clínica deste paciente, também dos sinais sintomas apresentados, diagnóstico médico e tratamento juntamente com assistência de enfermagem responder a seguinte questão norteadora: Quais os cuidados de enfermagem frente a atenção ao paciente com suspeita de câncer cabeça e pescoço.

MÉTODO

O relato de experiência foi vivenciado durante as atividades como enfermeiras residentes no setor de uma Unidade de Internação Clínica, ao longo da atuação no curso de Pós-Graduação em nível de especialização em Clínica Médica e Cirúrgica sob a forma de treinamento em serviço.

O presente trabalho trata-se de um estudo descritivo, de abordagem qualitativa, do tipo relato de experiência. Para a realização do estudo e coleta dos dados foram utilizadas as informações do prontuário do próprio paciente, realizando ainda, anamnese e histórico de enfermagem. 
Para garantir segurança dos dados colhidos e garantia de não identificação do usuário, foi assinado o Termo de Consentimento Livre Esclarecido (TCLE) pelo familiar responsável, este respeitando os preceitos Éticos de Pesquisa com Seres Humanos, fundamentado na Resolução 466/2012 do Conselho Nacional de Saú$\mathrm{de}^{10}$. Estudo este, aprovado pelo Comitê de Ética em Pesquisa da Instituição com o seguinte número de parecer: 4.439.007.

O levantamento dos dados do paciente utilizado nos resultados e para a realização dos diagnósticos de enfermagem e evidenciação dos cuidados de enfermagem para o seguinte caso, sendo fundamentado na experiência e vivência de residentes de enfermagem no período em que atuaram em uma Unidade de Internação Clínica de um Hospital Federal da cidade do Rio de Janeiro. Em relação ao tempo de pesquisa, coleta, escrita e finalização, ocorreu no período de agosto a outubro do ano de 2020 .

\section{RESULTADOS}

O presente relato descreve a vivência de residentes acrescidas a pesquisa realizada através do prontuário e da anamnese realizada com o paciente, apresentado no estudo de caso a seguir:

Paciente masculino, morador do estado do Rio de Janeiro, 88 anos, 2 fiIhos, chegou na emergência no dia 15/08/2020, acompanhado pela esposa. Apresentando história de disfagia, afasia e rouquidão há mais ou menos 10 dias. Histórico de emagrecimento, etilismo, tabagismo, hipertensão arterial sistêmica, diabetes mellitus, mobilidade e força de membros reduzidos, nega alergia medicamentosa. Internado no setor Unidade de Internação Clínica no dia 17/08/2020, onde foi realizado laringoscopia, endoscopia digestiva alta (EDA), tomografia de crânio nos dias 15/08, 18/08 e 23/08 de agosto. Possível broncoaspiração em 30/08, transferido ao Centro de Terapia Intensiva em 31/08, realizado acesso venoso profundo (PVF) em femoral, cateter vesical de demora (CVD) e cateter nasoenteral (CNE), permaneceu no centro por 11 dias, aguardando gastrostomia. Retornou para Unidade de Internação Clínica em 10/09, com diagnóstico base inconclusivo e suspeita de neoplasia de cabeça e pescoço. O paciente veio a óbito em dia 17/09.

O tema abordado foi escolhido, devido à dificuldade de esclarecimento do diagnóstico, e a falta de registros no prontuário. Visto que sem o diagnóstico correto, há uma dificuldade relacionado a clínica.

Neste setor, há um quantitativo elevado de pacientes oncológicos; é uma experiência, além de estressante, emocionante, e dolorosa, porque um simples toque, um simples gesto, já se percebe a reação do paciente, os sentimentos por ele expressados, observamos o quanto é importante para ele naquela fase tão difícil, mas este paciente em especial, chamou atenção, pelo seu olhar que demonstrava precisar de ajuda. Seu essencial mecanismo encontrava-se prejudicado, a sua comunicação. Diante de tudo, encontrava-se longe de seus familiares, em ambiente hospitalar, exposto a procedimentos invasivos muitas vezes com seu emocional fragilizado.

A morte é um fenômeno que ocorre no dia a dia da equipe de saúde, contudo sempre será de difícil enfrentamento. O medo e insegurança para lidar com a finitude, juntamente com sentimento de impotência, fizeram parte desta fase, contudo a assistência de enfermagem foi realizada, com um olhar holístico, sempre ofertando medidas de conforto.

\section{DISCUSSÃO}

Ao analisar os dados coletados do paciente, foi possível ter uma visão holística perante as suas necessidades apresentadas, o que possibilitou o entendimento, a prescrição de Intervenções de Enfermagem e o planejamento da assistência para o seu caso em especifico, pois segundo Berwanger et al (2019)11 "Quan- do a assistência é planejada, é possível diagnosticar as necessidades do cliente, favorecendo uma prescrição adequada dos cuidados de enfermagem".

O paciente apresentando de forma significativa uma comunicação prejudicada e dificuldade na escrita, fazia suas solicitações através dos sinais, gesticulações e olhares. Quando um dos principais recursos do paciente consta como prejudica, como a comunicação verbal, deve-se realizar adequações por parte de toda equipe ao longo de todo o processo do cuidado, sendo pertinente aos profissionais de enfermagem para manter o vínculo, ganhar confiança e conseguir realizar todas as etapas do processo de enfermagem ${ }^{12}$.

A partir do momento em que é aplicado a comunicação terapêutica é relevante que o enfermeiro fique atento para os recursos verbais e não verbais da linguagem, estes mecanismos constituem o aspecto técnico. Contudo é fundamental que o profissional conheça o contexto social, econômico e psicológico vivenciado pelo paciente, pois existem maneiras diferentes e individuais de vivenciar determinadas situações, estes fatores possibilitam que a comunicação seja um ato humano ${ }^{12}$.

Segundo o Instituto Nacional do Câncer os tipos de câncer mais frequente em homens, à exceção do câncer de pele não melanoma serão próstata $(29,2 \%)$, colón e reto $(9,1 \%)$, pulmão $(7,95)$, estômago $(5,9 \%)$ e cavidade oral $(5,0 \%)$. A distribuição da incidência por Região geográfica mostra que a Região Sudeste concentra mais de $60 \%$ da incidência, seguida pelas Regiões Nordeste $(27,8 \%)$ e Sul $(23,4 \%)$. Existe, entretanto, grande variação na magnitude e nos tipos de câncer entre as diferentes Regiões do Brasil ${ }^{13}$.

Diante das disposições da lei $\mathrm{N}^{\circ}$ 7.498, de 25 de junho de 1986 e do decreto $n^{\circ} 94406$ de 08 de julho de 1987, que regulamentar, cabe ao enfermeiro a liderança, na execução e avaliação do processo de enfermagem, de modo a alcançar os resultados de enfermagem es- 
perados, cabendo the privativamente, o diagnóstico de enfermagem acerca das respostas do paciente, família ou coletividade humana em um dado momento do processo de saúde e doença, bem como prescrição das ações ou intervenções de enfermagem a serem realizadas, face a essas respostas ${ }^{14}$.

Os enfermeiros têm o compromisso de assegurar a qualidade da assistência proporcionado, carecendo, incentivando e alertando toda a equipe a executar cuidados de enfermagem com qualidade. É fundamental que a equipe esteja empenhada as necessidades dos pacientes e suas dificuldades para lidarem com os desafios do dia a dia, sendo capaz de auxiliar nos momentos de impaciência que podem surgir ${ }^{15}$.

A identificação precoce do câncer é um aspecto determinante do prognóstico do CCP. O Instituto Nacional do câncer certifica que o seu intuito é estimular a vigilância da doença, apontando as estimativas para cada ano do triênio 2020 - 2022 com veracidade, para se instituir um instrumento a ser usado por gestores, profissionais da saúde, bem como pela sociedade em geral como forma de implementação das condutas de promoção a saúde e prevenção da doença ${ }^{13}$.

\section{DIAGNÓSTICO E INTERVENÇÕES DE ENFERMAGEM}

Após coleta dos dados e realização da anamnese, seguiu-se o processo de Enfermagem, realizando o planejamento, diagnóstico e intervenções selecionados conforme as necessidades especificas do caso e do quadro clinico, evidenciando assim, os cuidados de enfermagem para paciente com suspeita de neoplasia de cabeça e pescoço, como descrito no Quadro 01.

Quadro 01: Diagnósticos, Resultados e Intervenções de Enfermagem para o caso.

\begin{tabular}{|c|c|c|}
\hline Diagnósticos & Resultados & Intervenções \\
\hline $\begin{array}{c}\text { Nutrição desequilibrada: menor do que } \\
\text { as necessidades corporais, relacionada: } \\
\text { a incapacidade de deglutir os alimentos, } \\
\text { caracterizada: pela por nódulos em região } \\
\text { cabeça e pescoço. }\end{array}$ & $\begin{array}{l}\text { Ingestão de alimentos e líqui- } \\
\text { dos por Cateter nasoenteral }\end{array}$ & $\begin{array}{l}\text {-Auxiliar na alimentação. } \\
\text {-Pesar o paciente em jejum diariamente. } \\
\text {-Realizar índice de massa corporal (IMC) }\end{array}$ \\
\hline $\begin{array}{c}\text { Padrão respiratório ineficaz relacionado } \\
\text { à energia diminuída caracterizado por } \\
\text { dispneia. }\end{array}$ & Equilíbrio dos sinais vitais & $\begin{array}{l}\text {-Manter cabeceira elevada }\left(30^{\circ}\right) \text {. } \\
\text {-Monitorizar frequência e ritmo respiratórios. } \\
\text {-Monitorizar gasometria arterial. } \\
\text {-Instalar oximetria de pulso. }\end{array}$ \\
\hline $\begin{array}{l}\text { Dor aguda, relacionada à: agente bioló- } \\
\text { gico e físico caracterizada por: sinais e } \\
\text { expressões faciais. }\end{array}$ & Controle da dor & $\begin{array}{l}\text {-Monitorar a intensidade da dor utilizando a escala de dor, a fim } \\
\text { de se ter uma mensuração de dor do paciente para posteriormente } \\
\text { estabelecer qual melhor conduta para tratá-la; } \\
\text {-Administrar analgésico, quando prescrito ou quando necessário } \\
\text { atentando para a prescrição no prontuário do paciente; } \\
\text {-Avaliar junto ao paciente, se a medicação está surgindo efeito sobre } \\
\text { a dor, para que possa comunicar a equipe médica sobre persistência } \\
\text { da dor, se caso houver. }\end{array}$ \\
\hline $\begin{array}{l}\text { Conhecimento deficiente relacionado à } \\
\text { falta de exposição, caracterizado por di- } \\
\text { ficuldade em verbalizar e entender sobre } \\
\text { sua patologia }\end{array}$ & $\begin{array}{l}\text { Conhecimento: cuidados na } \\
\text { doença. }\end{array}$ & $\begin{array}{l}\text {-Avaliar capacidade cognitiva do paciente. } \\
\text {-Orientar o paciente quanto a sua doença. } \\
\text {-Ensinar e incentivar o autocuidado. } \\
\text {-Esclarecer dúvidas do paciente quanto a doença. }\end{array}$ \\
\hline
\end{tabular}




\begin{tabular}{|c|c|c|}
\hline Risco de aspiração & $\begin{array}{c}\text {-Prevenir bronco aspiração } \\
\text {-Prevenir complicações }\end{array}$ & $\begin{array}{l}\text {-Monitorar nível de consciência, reflexo de tosse, náusea e capacida- } \\
\text { de de deglutir } \\
\text {-Deixar avisado para equipe que o paciente tem risco de broncoaspi- } \\
\text { ração. Não ofertar água. } \\
\text {-Posicionar o paciente em decúbito de } 45^{\circ} \text {, no mínimo } \\
\text {-Manter a cabeça do paciente lateralizada, quando recomendado } \\
\text {-Manter aspirador disponível }\end{array}$ \\
\hline Risco de infecção & & $\begin{array}{l}\text {-Monitorar sinais e sintomas de infecção (rubor, calor, edema, } \\
\text { hiperemia) } \\
\text {-Higienizar as mãos com gel alcoólico antes e depois de cada } \\
\text { procedimento. } \\
\text {-Fixar os acessos venosos periféricos e profundos com filme estéril } \\
\text { para facilitar a visualização do óstio e inserção do cateter. }\end{array}$ \\
\hline
\end{tabular}

\section{CONCLUSÃO}

Mesmo diante da dificuldade do diagnóstico os cuidados prestados foram de qualidade e excelência. Entende-se que o planejamento das ações por meio da Sistematização da Assistência de Enfermagem (SAE) é imprescindível para o pensamento crítico e reflexivo, humanizado pela equipe quanto aos cuidados a serem prestados aos pacientes.

A presença de fatores estressores nos pacientes acometidos pelo câncer cabeça e pescoço faz refletir sobre o papel do enfermeiro no cuidado desses pacientes,

\section{S6}

$$
\begin{aligned}
& \text { Mesmo diante da } \\
& \text { dificuldade do } \\
& \text { diagnóstico os } \\
& \text { cuidados prestados } \\
& \text { foram de qualidade } \\
& \text { e excelência. }
\end{aligned}
$$

observando a importância de realizar cuidados específicos baseados na necessidade de cada paciente, buscando melhora do estudo e garantindo maior conforto durante a permanência na unidade hospitalar.

Em vista disso, conclui-se que a assistência de enfermagem ao paciente com câncer cabeça e pescoço são essenciais para a qualidade do cuidado e para sua efetiva recuperação. Sendo um relato de experiência, permite apresentar sentimentos vivenciados, entender melhor as dificuldades e identificar quais os cuidados a serem prescritos e realizados.

\section{Referências}

1. Rio de Janeiro. Ministério da Saúde. Ministério da Saúde. Instituto Nacional do Câncer Ministério da Saúde.

2. Ministério da Saúde Instituto Nacional de Câncer. Estimativa 2020: Incidência de Câncer no Brasil - Rio de Janeiro: INCA, 2019.

3. Rio de Janeiro. Ministério da Saúde. Ministério da Saúde. Instituto Nacional do Câncer Ministério da Saúde.

4. Sociedade Brasileira de Oncologia Clínica SBOC (São Paulo). Sociedade Brasileira de Oncologia Clínica SBOC: câncer de cabeça e pescoço. Câncer de Cabeça e Pescoço. 2016.

5. Oliveira JM, Reis JB, Silva RA. Busca por cuidado oncológico: percepção de pacientes e familiares. Revista de Enfermagem Ufpe On Line, v. 12, n. 4, p. 938-946, 4 abr. 2018.

6. Sociedade Brasileira de Oncologia Clínica SBOC (São Paulo). Sociedade Brasileira de Oncologia Clínica SBOC: câncer de cabeça e pescoço. Câncer de Cabeça e Pescoço, 2016.

7. Costa MCM, Melo CF, Baião DC, Cavalcante AKS. Communication of a new message: The diagnosis of cancer in the perspective of patients and professionals. J Nurs UFPE on line Recife. (Suppl 8), p. 3214-3221.

8. Nanda-I. Diagnósticos de enfermagem da NANDA: definições e classificação 2015/2017. Porto Alegre, Artmed, 2015.
9. Silva MJP. Comunicação de Más Notícias: o mundo da saúde. 0 Mundo da Saúde, São Paulo, p. 49-53, 03 jan., 2012.

10. Resolução $n^{\circ}$. 466, de 12 de dezembro de 2012 (BR). Dispõe sobre Pesquisa envolvendo Seres Humanos. Diário Oficial da União, Brasília (DF). 11. Berwanger DC, et al. Processo de enfermagem: vantagens e desvantagens para a prática clínica do enfermeiro. Nursing (Säo Paulo), v. 22, n. 257, p. 3204-3208, out., 2019.

12. Silva RC, Barros CVL. Comunicação Terapêutica Relacionada ao Cuidado Humanizado e a Segurança do Paciente em Unidade Hospitalar. Revista Acadêmica do Instituto de Ciências da Saúde: Saúde \& Ciência em Ação, São Paulo, p. 13-25, 01 jul. 2015.

13. Instituto Nacional de Câncer José Alencar Gomes da Silva. Estimativa 2020: Incidência de Câncer no Brasil - Rio de Janeiro: INCA, 2019.

14. Conselho Federal de Enfermagem. Resolução COFEN-272/2002 - Revogada pela Resolução COFEN n 358/2009. Normatiza o histórico (entrevista), exame físico, diagnóstico, prescrição e evolução de enfermagem. .... Artigo $2^{\circ}$ - A implementação da Sistematização da Assistência de Enfermagem. 15. Silva CN, et al. Exercício da Liderança do(A) Enfermeiro(A) em unidades oncológicas. Revista Baiana de Enfermagem, Salvador, v. 30, n. 2, p.1-10, abr. 2016. 